\title{
miR-338 inhibits the metastasis of lung cancer by targeting integrin $\beta 3$
}

\author{
XIAO CHEN $^{1}$, LI WEI $^{2}$ and SONG ZHAO ${ }^{1}$ \\ ${ }^{1}$ Department of Thoracic Surgery, The First Affiliated Hospital of Zhengzhou University, Zhengzhou, Henan 450003; \\ ${ }^{2}$ Department of Thoracic Surgery, The People's Hospital of Henan Province, Zhengzhou, Henan 450003, P.R. China
}

Received February 20, 2016; Accepted May 27, 2016

DOI: 10.3892/or.2016.4928

\begin{abstract}
R-338 as an intronic miRNA from apoptosisassociated tyrosine kinase (AATK) is involved in tumor proliferation and apoptosis, but its function and regulatory mechanism in lung cancer is still obscure. In the present study, we found that miR-338 was strikingly downregulated in 115 lung cancer tissues and 5 lung cancer cell lines. Besides, low level of miR-338 was associated with tumor emboli, TNM stage, tumor recurrence and poor survival. Regaining the expression of miR-338 in lung cancer cell lines significantly impaired cellular adhesion, migration, invasion and lung tumor formation in nude mice. Furthermore, we also identified a metastasis related protein, integrin $\beta 3$ (ITGB3), as a novel target gene of miR-338. Our results reveal a new regulatory mechanism of miR-338 which may help us better understand the metastasis of lung cancer.
\end{abstract}

\section{Introduction}

Lung cancer is the leading cause of cancer associated death all over the world (1). Tumor metastasis as the major cause for high mortality of lung cancer has attracted a great deal of research interest. During the past decades, many metastasis related processes had been identified, such as genetic mutation (2), angiogenesis (3), drug resistance (4), inflammation (5), cancer stem cells (6) and epithelial-mesenchymal transition (EMT) (7). In addition, understanding the molecular mechanism of metastatic lung cancer has greatly improved patient survival.

ITGB3 is a receptor of various proteins such as fibronectin, laminin, matrix metalloproteinase-2, osteomodulin and vitronectin (8). Highly elevated expression of ITGB3 has been observed in various kinds of malignant carcinoma. In leukemia, ITGB3 plays a crucial role in leukemogenesis,

Correspondence to: Professor Song Zhao, Department of Thoracic Surgery, The First Affiliated Hospital of Zhengzhou University, Zhengzhou, Henan 450003, P.R. China

E-mail: zhaosong@zzu.edu.cn

Key words: lung cancer, miRNAs, miR-338, ITGB3, metastasis making ITGB3 be a potential therapeutic target in AML (9). Another study confirmed that ITGB3 was an important regulator in reactive oxygene species induced migration and invasion of colorectal cancer cells (10). In breast cancer, mRNA profile array revealed that several angiogenesis related proteins including ITGB3 were significantly upregulated in metastatic tumor cells (11). Moreover, a recent study proved that let-7c, which is downregulated in lung cancer, inhibited the migration and invasion of lung cancer cells by targeting ITGB3 (12).

MicroRNAs (miRNAs) are non-coding RNA, 20-22-nucleotides in length and can regulate gene expression by repressing gene translation or promoting mRNA degradation (13). Disordered expression of miRNAs play an important role in tumor initiation, progression and recurrence. Some miRNAs such as miR-92b (14), miR-9 (15), miR-224 (16) and miR-183 (17) act as oncogenes and drive tumor metastasis in lung cancer. While, miR-101 (18), miR-133a (19) and miR-141 (20) which are downregulated in lung cancer could significantly suppresses tumor metastasis. Besides, some exosomal miRNAs play a key role in lung carcinogenesis, making miRNAs new tumor biomarkers (21).

miR-338 was firstly identified as an intronic miRNA of its host gene AATK and was also functionally antagonistic to AATK (22). Recently, miR-338 was proved downregulated in hepatocellular carcinoma (23), oral carcinoma and esophageal squamous cell carcinoma (24). In gastric cancer, overexpressing miR-338 inhibited cell proliferation and promoted apoptosis (25). Restoring miR-338 level in hepatocarcinoma sensitized cells to sorafenib (26). Although one study had proved that miR-338 was able to inhibit colorectal cancer cell invasion and migration by targeting Smoothened (27). The mechanism of miR-338 in tumor metastasis is still unclear. In the present study, we assessed the expression of miR-338 in 115 pairs of lung cancer by real-time PCR assay. We also attempted to clarify the function and molecular mechanism of miR-338 in lung cancer metastasis.

\section{Materials and methods}

Cell culture and patients samples. Five lung tumor cell lines A549, NCI-H292, NCI-H460, NCI-H446, NCI-H1299 and one human lung fibroblast cell MRC-5 were purchased from the Cell Bank of Shanghai. All cells were maintained in 
Dulbecco's modified Eagle's media (DMEM) or RPMI-1640 medium supplemented with $10 \%$ fetal bovine serum (FBS; Life Technologies, Carlsbad, CA, USA), $100 \mathrm{U} / \mathrm{ml}$ penicillin and $100 \mathrm{U} / \mathrm{ml}$ streptomycin. A549 or NCI-H292 miR-338 overexpressing cell lines were constructed by lentivirus assay (Shanghai Genechem, Co., Ltd., Shanghai, China). Human lung cancer and adjacent normal tissues were obtained from 115 patients in the First Affiliated Hospital of Zhengzhou University. All samples were obtained with written patient informed consent and the study was approved by the Medical Ethics and Human Clinical Trial Committee.

RNA isolation and real-time quantitative PCR. Total RNA from 115 pairs of lung cancer tissues or cell lines were extracted with TRIzol (Invitrogen, Carlsbad, CA USA) and the small non-coding miRNAs were isolated by mirVana miRNA Isolation kit (Ambion, Austin, TX, USA) according to the manufacturer's instruction. A total of $1 \mu \mathrm{g}$ miRNAs were reverse transcripted with Mir- $\mathrm{X}^{\mathrm{TM}}$ miRNA First-Strand Synthesis kit (Takara Bio, Beijing, China) and the expression of mR-338 was detected with Mir- ${ }^{\mathrm{TM}}$ miRNA qRT-PCR SYBR kit (Takara Bio). Primers for miR-338 and U6 snRNA were also purchased from Takara Bio.

CCK-8 proliferation assay. In order to examine the effect of miR-338 on cellular proliferation, miR-338 overexpressing or miR-control cells were seeded on 96 -well plates $(2,000 /$ well $)$. Then, $10 \mu \mathrm{l}$ Cell Counting kit-8 (CCK-8) was added for $1 \mathrm{~h}$ at $37^{\circ} \mathrm{C}$. The absorbance of OD450 was measured by microplate reader (Bio-Rad Laboratories, Hercules, CA, USA), with OD630 as a reference wavelength. All the experiments were repeated three time in triplicate.

Cell adhesion assay. Cell adhesion assay was performed to assess the effect of miR-338 on cellular adhesion. Firstly, the 24-well plates (Corning, Inc., Corning, NY, USA) were coated with matrix gel (1:50 dilution; BD Biosciences, San Jose, CA, USA) overnight at $4^{\circ} \mathrm{C}$. Then, $1 \times 10^{5} \mathrm{miR}-338 / \mathrm{miR}$-control stable infected cells were seeded on the plates, and incubated for $30 \mathrm{~min}$ at $37^{\circ} \mathrm{C}$. The suspended cells were washed out with PBS twice, and adhesion cells were fixed with $4 \%$ paraformaldehyde. Finally, cells were stained with $0.4 \%$ typan blue, photographed and counted under a microscope.

Transwell migration and invasion assay. Transwell migration assays were performed with 24-well Transwell plates (Corning). Lung cancer cells $\left(5 \times 10^{4}\right)$ suspended in $100 \mu \mathrm{l}$ serum-free medium were added into the upper chamber of plates, and $500 \mu 1$ medium with $10 \%$ FBS was added into the lower chamber. After $12 \mathrm{~h}$, the upper chamber was fixed, cells on the inner layer were removed with a cotton swabs, stained with $0.4 \%$ typan blue and counted at x100 magnification. For invasion assay, $1 \times 10^{5}$ cells were seeded on matrix gel (BD Biosciences) pre-coated Transwell chamber, then following the procedure of migration assay.

Xenograftumor model. Six pairs of 5-weeks old femal BALB/c nude mice (Vital River Laboratory Animal Technology, Co., Ltd., Beijing, China) were implanted with $1 \times 10^{6}$ NCI-H292 miR-control or miR-338 cells in $100 \mu 1$ PBS by lateral tail vein injection. Two months later, all animals were sacrificed and the lung were dissected and fixed with $4 \%$ paraformaldehyde. Then the tissues were sectioned and stained with hematoxylin and eosin (H\&E). Furthermore, the number of tumor clones in the lungs were counted.

Dual-luciferase activity assay. Lung cancer cells $\left(1 \times 10^{4}\right)$ seeded in 24-well plates were co-transfected with $50 \mathrm{ng}$ ITGB3 wild-type 3'UTR (ITGB3-3'UTR-WT)/ITGB3 mutant 3'UTR (ITGB3-3'UTR-MUT) (GeneCopoeia, Guangzhou, China), $100 \mathrm{nM}$ miRNA control/miR-338 mimics (Shanghai GenePharma, Co., Ltd., Shanghai, China), and 10 ng Renilla luciferase vector (Promega, Madison, WI, USA) with Lipofectamine 3000 (Life Technologies). Cells were harvested for luciferase activity assay at $48 \mathrm{~h}$ after transfection using Dual-luciferase reporter assay kit (Promega). All experiments were performed independently in triplicate.

Western blot analysis. Cells were lysed in RIPA lysis buffer (Beyotime Institute of Biotechnology, Haimen, China) supplemented with protease inhibitor mix (Roche Diagnostics, Indianapolis, IN, USA). Equal amount of protein was separated by $8 \%$ SDS-PAGE and then transferred to PVDF membranes (Millipore, Billerica, MA, USA). Membranes were blocked by $5 \%$ non-fat milk for $1 \mathrm{~h}$, incubated with ITGB3 antibody (Cell Signaling Technology, Danvers, MA, USA) or $\beta$-actin antibody (Zhongshan Bio, Co., Ltd., Zhongshan, China) overnight, then incubated with HRP-conjugated secondary antibody and detected by Pierce ${ }^{\mathrm{TM}}$ ECL Plus western blotting substrate kit (Thermo Fisher Scientific, Waltham, MA, USA).

Statistical analysis. Data were analyzed and presented as the mean \pm standard deviation (mean \pm SD) using SPSS 13.0. Difference between two group were estimated with Pairedsamples t-test. The association between miR-338 expression and clinicopathological factors were analyzed with one-way ANOVA and survival curves were plotted according to the Kaplan-Meier method using log-rank test. Difference between groups were considered statistically significant at $\mathrm{P}<0.05$.

\section{Results}

miR-338 was downregulated in lung cancer cell lines. We firstly analyzed the gene location of miR-338 in Gene Database. As shown in Fig. 1A, miR-338 was located in the seventh intron of AATK gene which was an adjacent gene of Brain specific angiogenesis inhibitor-associated protein 2 (BAIAP2). Studies had demonstrated BAIAP2 was an important adaptor protein that links Rho-family small GTPases, and was involved in cell motility and the reorganization of actin cytoskeleton (28-30). Considering adjacent genes often has similar or reverse function $(31,32)$, we inferred that miR-338 may participate in the progress of angiogenesis or cell motility.

Then, we analyzed miR-338 expression in normal human lung fibroblast MRC-5 and five lung cancer cell lines. From the results we found that miR-338 was evidently repressed in all lung cancer cell lines compared with MRC cells (Fig. 1B).

The expression and clinical significance of $m i R-338$ in lung cancer tissues. In order to further confirm the level of miR-338 


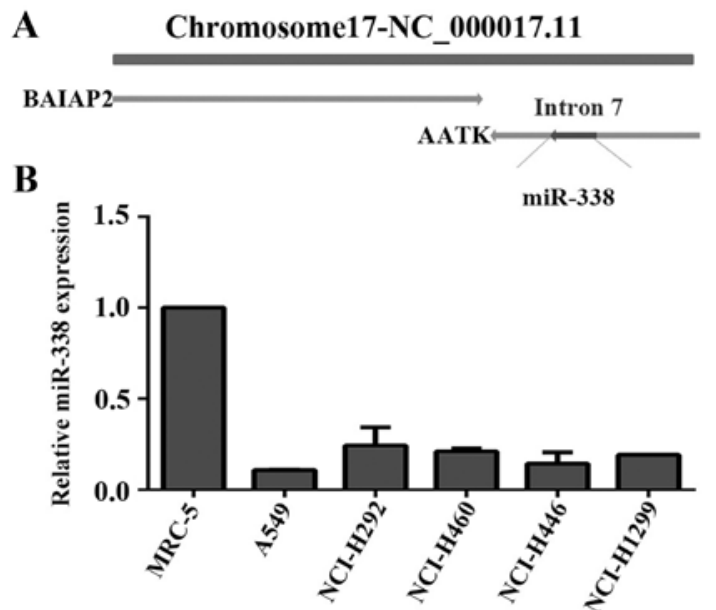

Figure 1. miR-338 is downregulated in lung cancer cell lines. (A) The gene location of miR-338 on the chromosome. (B) The relative expression of miR338 in 5 lung cancer cell lines compared with normal human lung fibroblast cells was examined by real-time PCR.

A
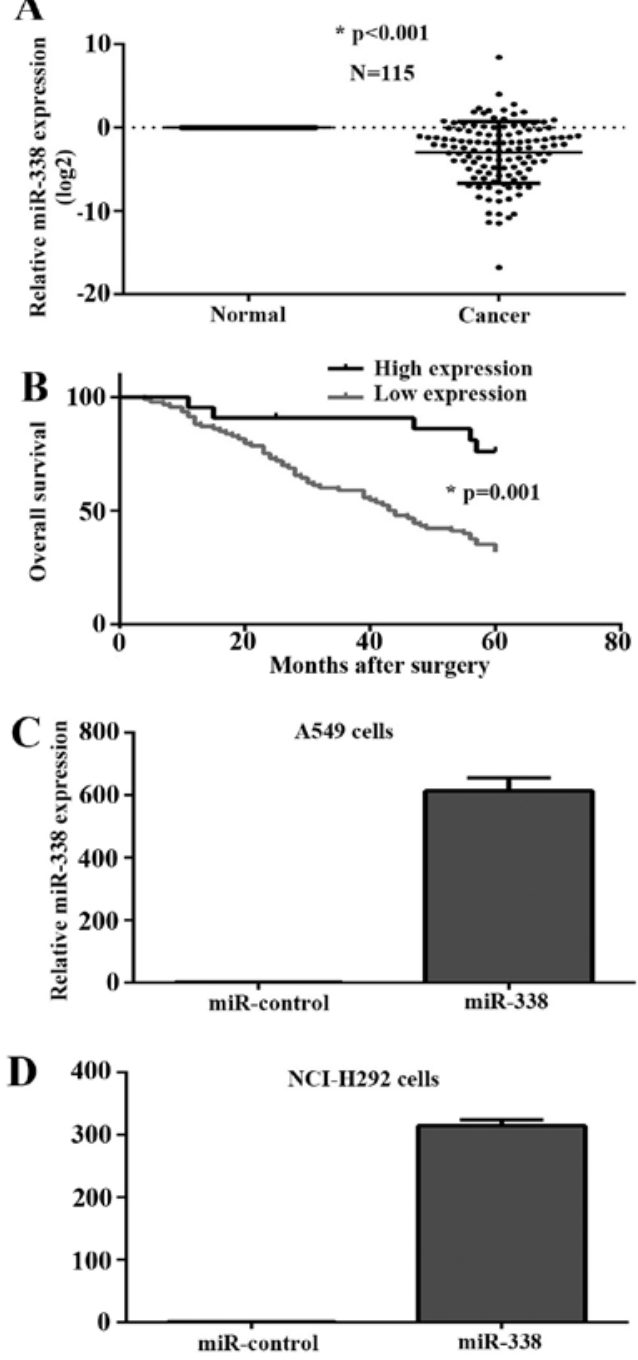

Figure 2. The expression and clinical significance of miR-338 in lung cancer tissues. (A) The expression of miR-338 in 115 pairs of lung cancer tissues was detected by real-time PCR. (B) The survival curves of patients with lung cancer based on miR-338 level, Kaplan-Meier method and log-rank test were used. (C) The expression of miR-338 in A549 overexpressing cell lines was assessed by real-time PCR assay. (D) The expression of miR-338 in NCIH292 overexpressing cell lines was assessed by real-time PCR assay.
Table I. The expression of miR-338 and clinocopathological factors in lung cancer.

\begin{tabular}{|c|c|c|c|}
\hline Factors & $\mathrm{N}$ & Relative expression & P-value \\
\hline \multicolumn{4}{|l|}{ Gender } \\
\hline Male & 87 & $-2.72 \pm 3.58$ & 0.236 \\
\hline Female & 28 & $-3.68 \pm 3.99$ & \\
\hline \multicolumn{4}{|c|}{ Age (years) } \\
\hline$<55$ & 45 & $-2.74 \pm 3.30$ & 0.618 \\
\hline$\geq 55$ & 70 & $-3.09 \pm 3.93$ & \\
\hline \multicolumn{4}{|l|}{ Smoking } \\
\hline Yes & 93 & $-2.84 \pm 3.72$ & 0.507 \\
\hline No & 22 & $-3.43 \pm 3.58$ & \\
\hline \multicolumn{4}{|c|}{ Tumor size (cm) } \\
\hline$\leq 3$ & 60 & $-2.45 \pm 3.69$ & 0.129 \\
\hline$>3$ & 55 & $-3.50 \pm 3.64$ & \\
\hline \multicolumn{4}{|c|}{ Tumor emboli } \\
\hline Yes & 26 & $-4.82 \pm 4.35$ & $0.005^{\mathrm{b}}$ \\
\hline No & 89 & $-2.44 \pm 3.44$ & \\
\hline \multicolumn{4}{|c|}{ Lymph node metastasis } \\
\hline Yes & 39 & $-3.28 \pm 3.52$ & 0.496 \\
\hline No & 76 & $-2.78 \pm 3.78$ & \\
\hline \multicolumn{4}{|c|}{ Tumor recurrence } \\
\hline Yes & 27 & $-4.72 \pm 4.23$ & $0.004^{\mathrm{b}}$ \\
\hline No & 88 & $-2.41 \pm 3.35$ & \\
\hline \multicolumn{4}{|l|}{ TNM } \\
\hline I-II & 65 & $-2.11 \pm 3.16$ & $0.025^{\mathrm{b}}$ \\
\hline III-IV & 50 & $-4.01 \pm 4.03$ & \\
\hline
\end{tabular}

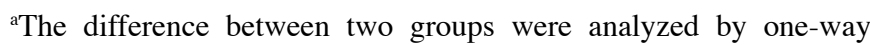
ANOVA. ${ }^{\mathrm{b}} \mathrm{P}<0.05$.

in lung cancer, we assessed the expression of miR-338 in 115 pairs of lung cancer using real-time quantitative PCR. Results demonstrated that miR-338 was significantly downregulated in tumor tissues (Fig. 2A). Moreover, patients with tumor emboli and recurrence had a lower expression of miR-338. Decreased miR-338 level was also associated with TNM stage, while there were no obviously difference about gender, age, smoking history, tumor size and lymph node metastasis (Table I). As shown in Fig. 2B, the 5-year overall survival rate of low miR-338 expressing group was significantly lower than that of the high miR-338 expressing group $(\mathrm{P}=0.001)$.

miR-338 inhibits the proliferation and adhesion of lung cancer cells. To investigate the function of miR-338 in the progress of lung cancer miR-338 overexpressing stable cell lines were constructed, and miR-338 RNA level was confirmed by real-time PCR assay (Fig. 2C and D). CCK-8 proliferation assay was performed to assess the effect of miR-338 on tumor growth. As shown in Fig. 3A and B, upregulation of miR-338 inhibited the proliferation rate. Furthermore, we decided to check whether miR-338 could affect cellular adhesion. Adhesion assay results revealed that overexpression of 

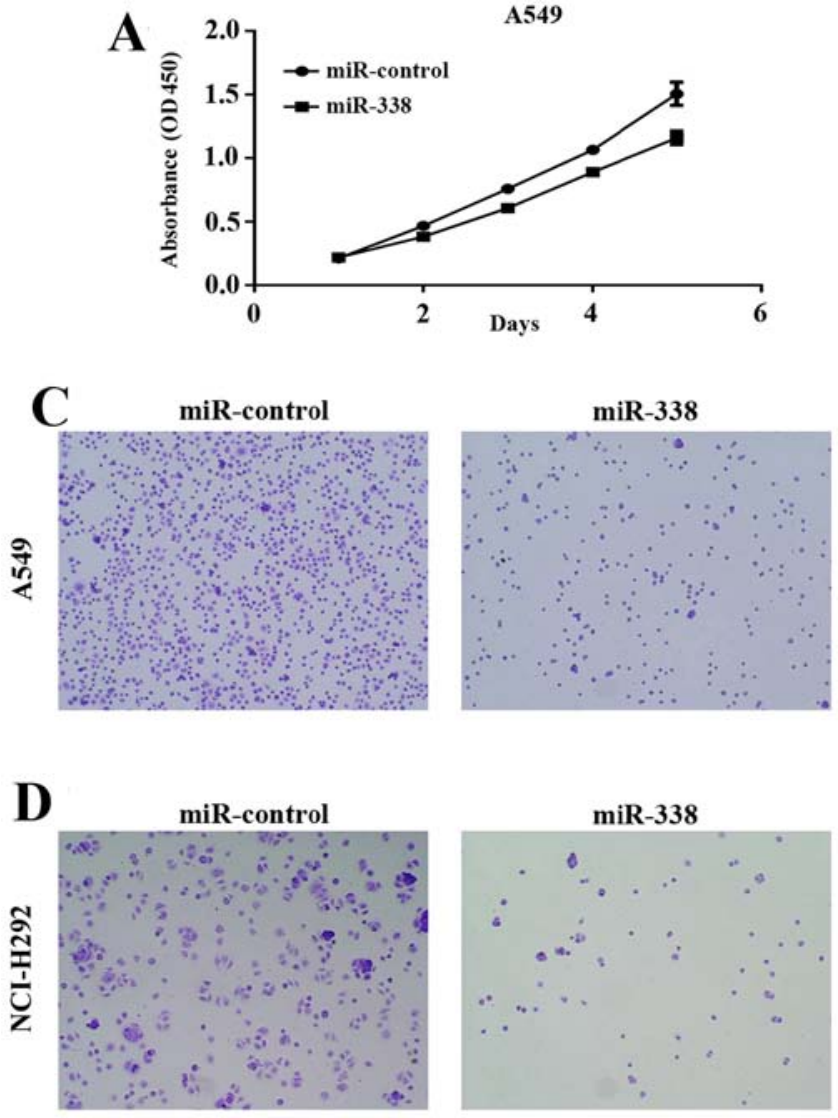

Figure 3. The effect of miR-338 on cellular proliferation and adhesion. (A) The viabilities of miR-338 overexpressing or miR-control A549 cells were determined using CCK-8 assay. (B) The viability of miR-338 overexpressing or miR-control NCI-H292 cells were determined using CCK-8 assay. (C) The effect of miR-338 on A549 cell adhesion ability were detected and the cell numbers were counted (magnification, x100). (D) The effect of miR-338 on NCI-H292 cell adhesion ability was detected and the cell numbers were counted (magnification, $\mathrm{x} 100$ ).

miR-338 in lung cancer cells obviously impaired the ability of adhesion (Fig. 3C and D).

miR-338 suppresses tumor migration and invasion. To explore the effect of miR-338 on cellular migration and invasion, Transwell assays were performed. Fig. 4A shows that elevating the expression of miR-338 in A549 and NCI-H292 cell lines markedly weakened the migration ability. This effect was also observed in the invasion assay (Fig. 4B).

miR-338 abated lung cancer metastasis in vivo. To further investigate whether miR-338 is sufficient to lighten tumor metastasis in vivo, miR-338 overexpressing and miR-control NCI-H292 cell lines were injected into the tail vein of BALB/c nude mice. Results shown that the miR-338 group had fewer tumor metastatic lesions compared with the control group (Fig. 5A). The tumor foci in lung tissues were confirmed by pathological section, and the tumor clone number was counted by microscope (Fig. 5B).

ITGB3 was a novel target gene of miR-338. To uncover the molecular mechanism by which miR-338 performed a suppressor role in cancer metastasis. The target genes were analyzed by software including StarBase, TargetScan and microRNA.org. Finally, we identified ITGB3 which was involved in tumor angiogenesis, cellular adhesion and cytoskeleton rearrangement as a potential target gene of miR-338
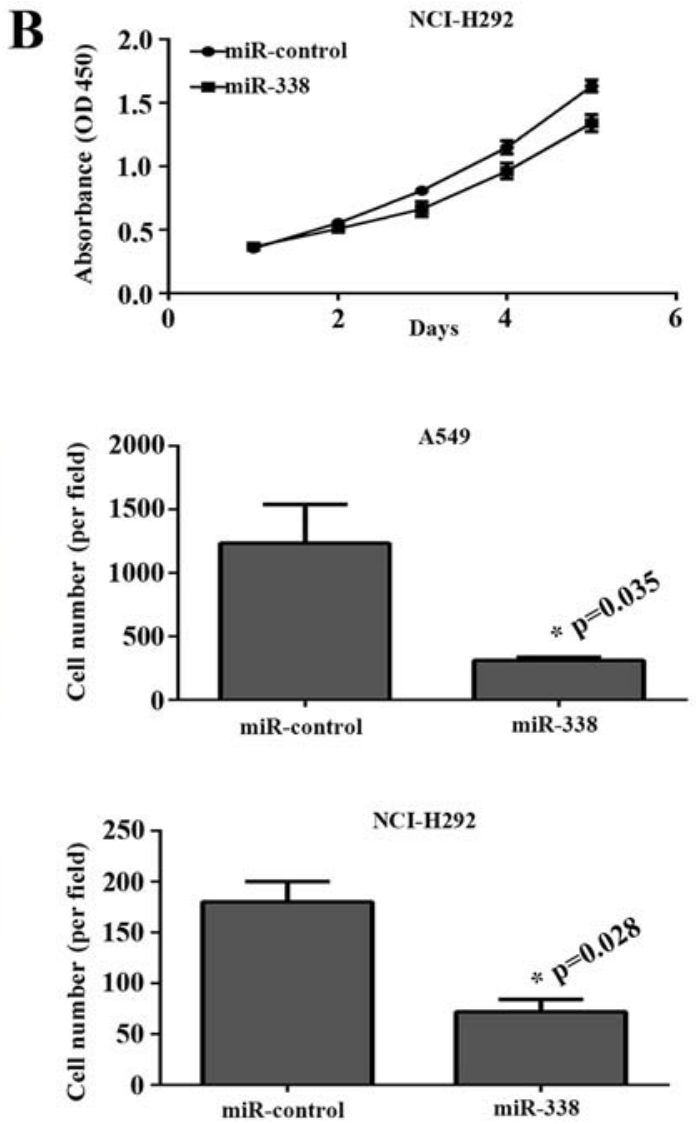

(Fig. 6A). Furthermore, we analyzed the correlation between miR-338 expression and ITGB3 mRNA expression in 441 lung cancer tissues from TCGA database. As shown in Fig. 6B, ITGB3 was negatively associated with miR-338 in lung cancer $(\mathrm{P}=0.0037)$.

To confirm this hypothesis, protein expression of ITGB3 in A549 and NCI-H292 miR-338 overexpressing stable cell lines were detected by western blot assay. The results revealed that exogenous transfection with miR-338 markedly suppressed ITGB3 expression (Fig. 6C). In order to examine whether ITGB3 was a direct target of miR-338, ITGB33'UTR-WT and ITGB3-3'UTR-MUT were constructed. Dual-luciferase activity assay revealed that the luciferase activity was significantly reduced in miR-338 mimics overexpressing group compared with the control group. However, this change was abrogated when the binding seed region was mutant (Fig. 6D and E).

\section{Discussion}

The role of miRNAs in the regulation of tumor metastasis has been widely recognized in the recent years. The progress on systemic delivery as well as applications for miRNAs as therapeutic agents have witnessed the advancement of miR-34a in liver cancer or metastatic cancer with liver involvement (33). Studies have proved that recovering some tumor suppressor miRNAs such as miR-204, miR-145, miR-21 significantly 

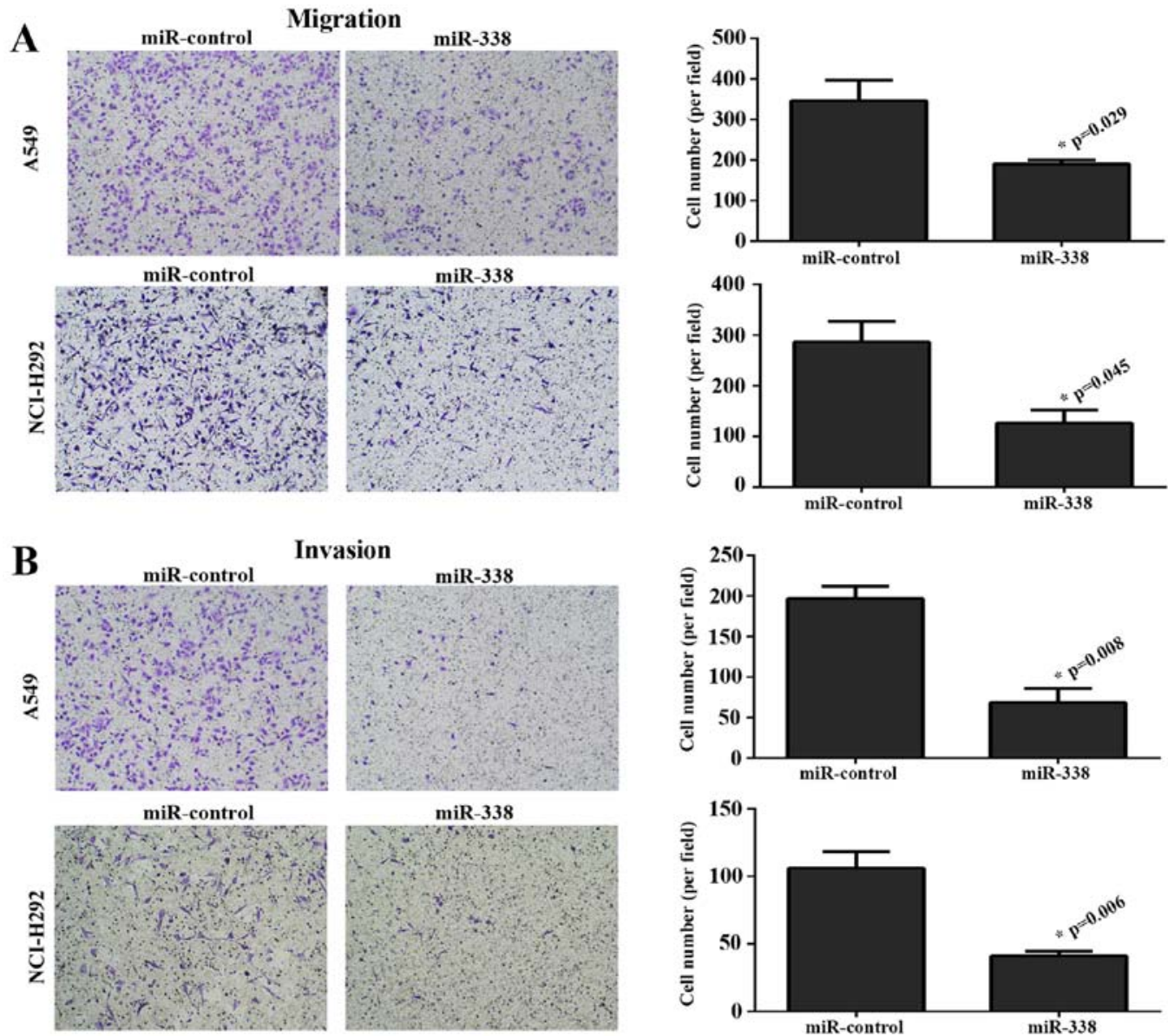

Figure 4. Impact of miR-338 on cell migration and invasion. (A) Migration assays were performed to investigate the migratory ability of A549 and NCI-H292 miR-388 overexpressing or miR-control cells, and the cell numbers were counted (magnification, $\mathrm{x} 100$ ). (B) Invasion assays were performed to investigate the invasive ability of A549 and NCI-H292 miR-388 overexpressing or miR-control cells, and the cell numbers were counted (magnification, x100).
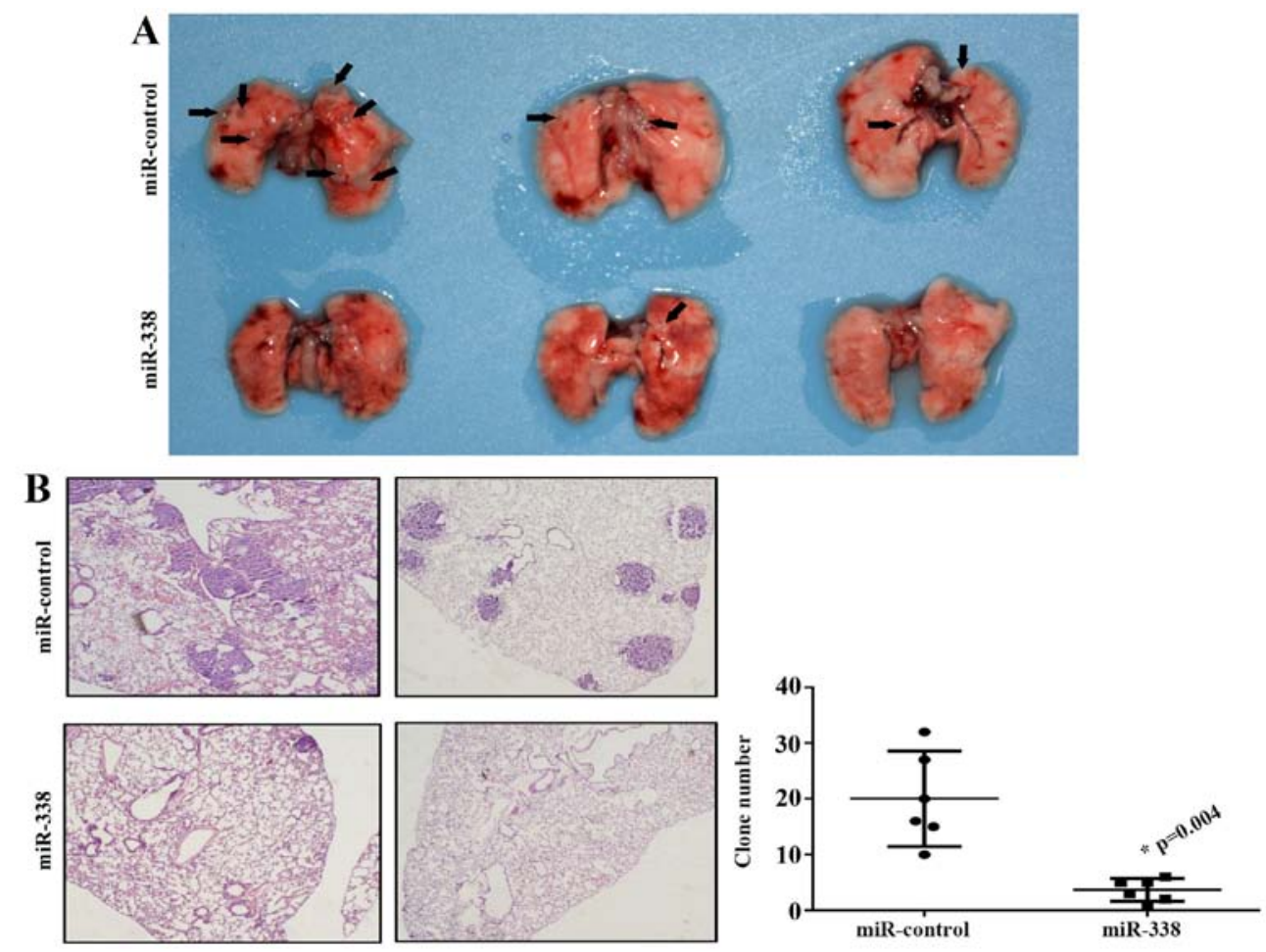

Figure 5. miR-338 abates lung cancer metastasis in vivo. (A) miR-338 overexpressing or miR-control NCI-H292 cells were injected into nude mice via the tail vein, lungs were dissected and photographed after 2 months. (B) Cancer cells in the lungs were confirmed by H\&E staining, the numbers of tumor clones were counted (magnification, $\mathrm{x} 100$ ). 
A

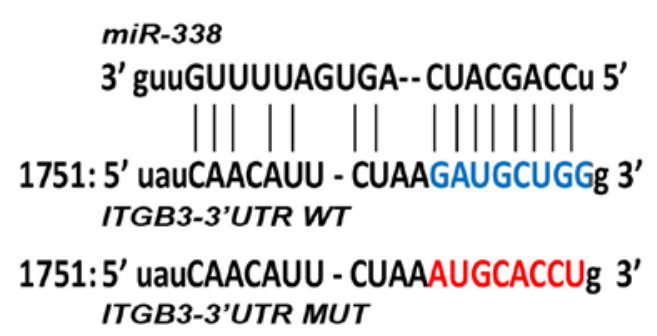

C
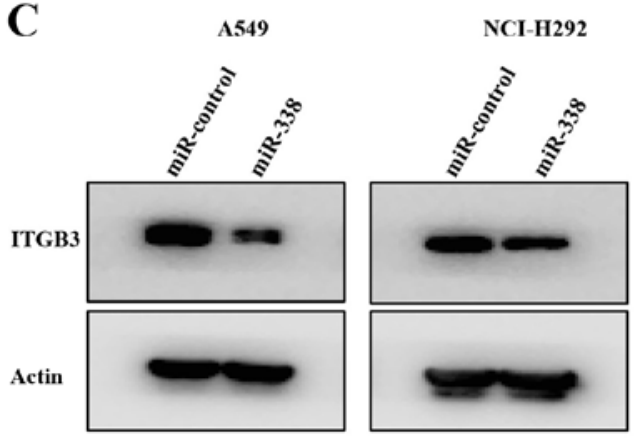

D

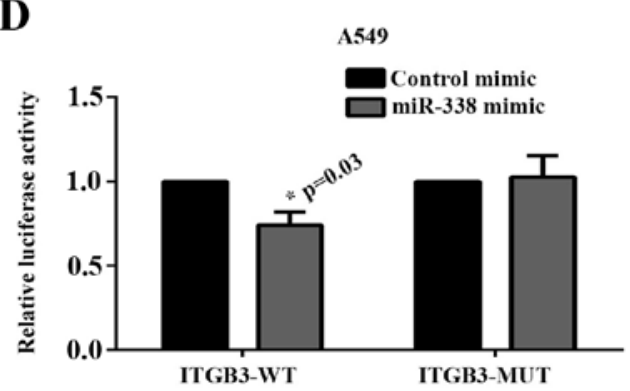

B
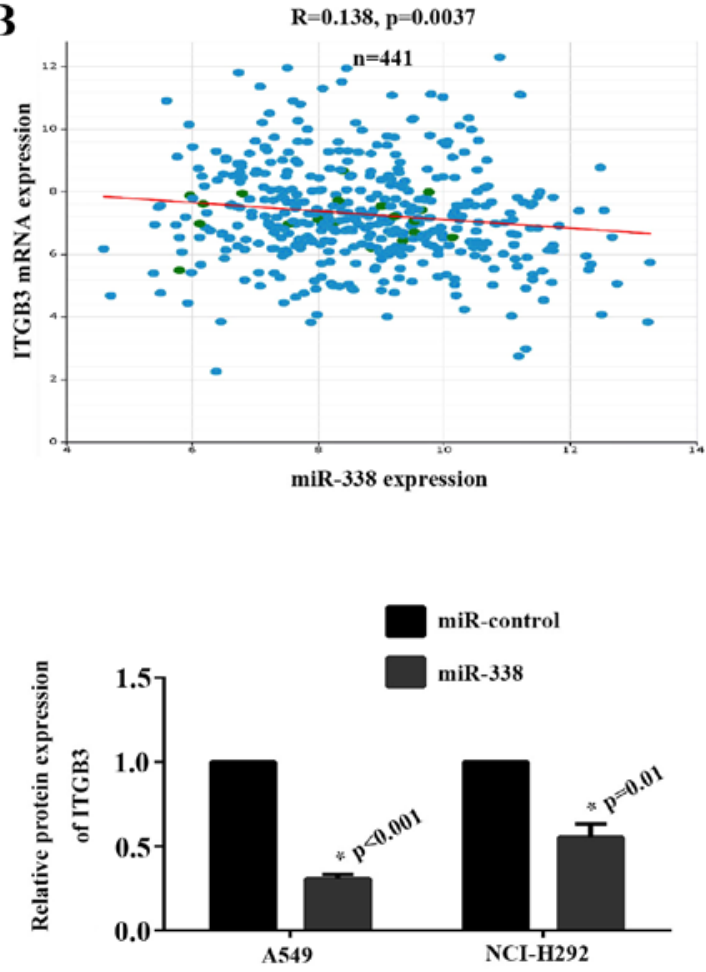

E

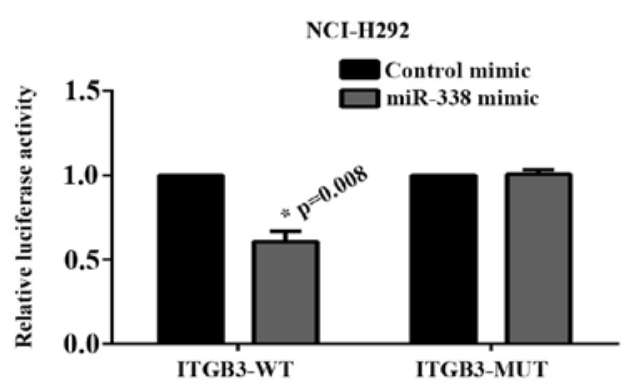

Figure 6. ITGB3 is a novel target gene of miR-338 in lung cancer. (A) The sequence of ITGB3 3'UTR-WT and ITGB3 3'UTR-MUT. (B) The correlation between miR-338 expression and ITGB3 mRNA expression in 441 lung cancer tissues from TCGA database. (C) The protein expression of ITGB3 in miR-338 overexpressing or miR-contrl cells, and the relative expression were analyzed by ImageJ. (D) The relative luciferase activity was determined by Dual-luciferase activity assay, when cotranfecting ITGB 3'UTR with miR-338 mimics or control mimics in A549 cells. (E) The relative luciferase activity was determined by Dual-luciferase activity assay, when cotransfecting ITGB 3'UTR with miR-338 mimics or control mimics in NCI-H292 cells.

abrogated the metastasis of human lung cancer in vitro and in vivo (34-36). Moreover, some plasma miRNAs including miR-486, miR-150, miR-152 and let-7c have been identified as biomarkers for lung cancer $(37,38)$. In the present study, we found that miR-338 was downregulated in lung cancer cell lines. We also validated that miR-338 was obviously impaired in lung cancer tissues. Lower expression of miR-338 was associated with tumor emboli and recurrence, indicating miR-338 may be a vital negative regulator of metastatic lung cancer.

AATK is a brain apoptosis-associated tyrosine kinase, and is a necessary pre-requisite for the induction of growth arrest, apoptosis and neuronal differentiation. Similar to host gene, miR-338 also displays its ability to regulate neurocyte differentiation or apoptosis (39-41). In carcinoma, miR-338 suppresses the proliferation of liver tumor cells by targeting foxp4, and induces gastric cancer apoptosis by targeting SSX2IP. The anti-proliferation effect of miR-338 was also confirmed in the present study. BAIAP2 as an adjacent gene of AATK is involved in neuronal growth-cone guidance, forma- tion of stress fibers and cytokinesis. As studies have validated that adjacent genes usually share similar or related function (42) besides AATK has also been identified as a regulator of neurite outgrowth (43). These findings hint that miR-338 may participate in cellular migration or invasion. Our further results validated that restoring the expression of miR-338 markedly impeded lung cancer cells metastasis and adhesion.

ITGB3 as a receptor of various extracellular matrix protein, and plays an important role in number of physiological and pathological progress such as bone resorption, angiogenesis, adhesion, tumor invasion and metastasis (44). Elevated expression of ITGB3 is closely correlated with the metastatic potential of colorectal cancer (10). In lung cancer, high expression of ITGB3 promotes tumor metastasis and vascular invasion (45). Studies have proven that some miRNAs including let-7a, let-7c and miR-320 inhibit tumor metastasis by targeting ITGB3 $(12,46,47)$. In this study, we identified that ITGB3 was a potential target gene of miR-338 through bioinformatics analysis. This was further confirmed by western blot 
assay and Dual-luciferase activity assay. We also found that ITGB3 was negatively correlated with miR-338 in 441 lung cancer tissues by analysing data from TCGA database.

In conclusion, our results show that miR-338 was downregulated in lung cancer and associated with tumor metastasis and recurrence. We also identified ITGB3, a tumor metastasis-related gene, as a novel target gene of miR-338, suggesting that miR-338 is a potential target for lung cancer therapy.

\section{References}

1. Siegel R, Ward E, Brawley O and Jemal A: Cancer statistics, 2011: The impact of eliminating socioeconomic and racial disparities on premature cancer deaths. CA Cancer J Clin 61: 212-236, 2011

2. Zhang X, Xiao D, Wang Z, Zou Y, Huang L, Lin W, Deng Q, Pan H, Zhou J, Liang C, et al: MicroRNA-26a/b regulate DNA replication licensing, tumorigenesis, and prognosis by targeting CDC6 in lung cancer. Mol Cancer Res 12: 1535-1546, 2014.

3. Hall RD, Le TM, Haggstrom DE and Gentzler RD: Angiogenesis inhibition as a therapeutic strategy in non-small cell lung cancer (NSCLC). Transl Lung Cancer Res 4: 515-523, 2015.

4. Liu MX, Zhou KC and Cao Y: MCRS1 overexpression, which is specifically inhibited by $\mathrm{miR}-129^{*}$, promotes the epithelialmesenchymal transition and metastasis in non-small cell lung cancer. Mol Cancer 13: 245, 2014.

5. Manraj K and Saurabh M: Chronic pain grade questionnaire. J Physiother 59: 60, 2013

6. Luanpitpong S, Li J, Manke A, Brundage K, Ellis E, McLaughlin SL, Angsutararux P, Chanthra N, Voronkova M, Chen YC, et al: SLUG is required for SOX9 stabilization and functions to promote cancer stem cells and metastasis in human lung carcinoma. Oncogene: Sep 21, 2015 (Epub ahead of print). doi: $10.1038 /$ onc. 2015.351 .

7. Jin Y, Li F, Zheng C, Wang Y, Fang Z, Guo C, Wang X, Liu H, Deng L, Li C, Wang H, et al: NEDD9 promotes lung cancer metastasis through epithelial-mesenchymal transition. Int $\mathbf{J}$ Cancer 134: 2294-2304, 2014.

8. Nurden AT, Fiore M, Nurden P and Pillois X: Glanzmann thrombasthenia: A review of $I T G A 2 B$ and $I T G B 3$ defects with emphasis on variants, phenotypic variability, and mouse models. Blood 118: 5996-6005, 2011.

9. Miller PG, Al-Shahrour F, Hartwell KA, Chu LP, Järås M, Puram RV, Puissant A, Callahan KP, Ashton J, McConkey ME, et al: In Vivo RNAi screening identifies a leukemia-specific dependence on integrin beta 3 signaling. Cancer Cell 24: 45-58, 2013.

10. Lei Y, Huang K, Gao C, Lau QC, Pan H, Xie K, Li J, Liu R, Zhang T, Xie N, et al: Proteomics identification of ITGB3 as a key regulator in reactive oxygen species-induced migration and invasion of colorectal cancer cells. Mol Cell Proteomics: MCP 10: M110 005397, 2011. doi: 10.1074/mcp.M110.005397.

11. Al-Sahaf O, Wang JH, Browne TJ, Cotter TG and Redmond HP: Surgical injury enhances the expression of genes that mediate breast cancer metastasis to the lung. Ann Surg 252: 1037-1043, 2010.

12. Zhao B, Han H, Chen J, Zhang Z, Li S, Fang F, Zheng Q, Ma Y, Zhang J, Wu N, et al: MicroRNA let-7c inhibits migration and invasion of human non-small cell lung cancer by targeting ITGB3 and MAP4K3. Cancer Lett 342: 43-51, 2014.

13. Bartel DP: MicroRNAs: Target recognition and regulatory functions. Cell 136: 215-233, 2009.

14. Lei L, Huang Y and Gong W: Inhibition of miR-92b suppresses nonsmall cell lung cancer cells growth and motility by targeting RECK. Mol Cell Biochem 387: 171-176, 2014.

15. Xu T, Liu X, Han L, Shen H, Liu L and Shu Y: Up-regulation of miR-9 expression as a poor prognostic biomarker in patients with non-small cell lung cancer. Clin Transl Oncol 16: 469-475, 2014.

16. Cui R, Meng W, Sun HL, Kim T, Ye Z, Fassan M, Jeon YJ, Li B, Vicentini C, Peng Y, et al: MicroRNA-224 promotes tumor progression in nonsmall cell lung cancer. Proc Natl Acad Sci USA 112: E4288-E4297, 2015.

17. Xu F, Zhang H, Su Y, Kong J, Yu H and Qian B: Up-regulation of microRNA-183-3p is a potent prognostic marker for lung adenocarcinoma of female non-smokers. Clin Transl Oncol 16 980-985, 2014
18. Yan F, Shen N, Pang J, Xie D, Deng B, Molina JR, Yang P and Liu S: Restoration of miR-101 suppresses lung tumorigenesis through inhibition of DNMT3a-dependent DNA methylation. Cell Death Dis 5: e1413, 2014.

19. Fujiwara T, Katsuda T, Hagiwara K, Kosaka N, Yoshioka Y, Takahashi RU, Takeshita F, Kubota D, Kondo T, Ichikawa H, et al: Clinical relevance and therapeutic significance of microRNA-133a expression profiles and functions in malignant osteosarcoma-initiating cells. Stem Cells 32: 959-973, 2014.

20. Lv M, Xu Y, Tang R, Ren J, Shen S, Chen Y, Liu B, Hou Y and Wang T: miR141-CXCL1-CXCR2 signaling-induced Treg recruitment regulates metastases and survival of non-small cell lung cancer. Mol Cancer Ther 13: 3152-3162, 2014.

21. Sun T, Kalionis B, Lv G, Xia S and Gao W: Role of exosomal noncoding RNAs in lung carcinogenesis. BioMed Res Int 2015: 125807, 2015.

22. Barik S: An intronic microRNA silences genes that are functionally antagonistic to its host gene. Nucleic Acids Res 36: 5232-5241, 2008

23. Recht M, Nemes L, Matysiak M, Manco-Johnson M, Lusher J, Smith M, Mannucci P, Hay C, Abshire T, O'Brien A, et al: Clinical evaluation of moroctocog alfa (AF-CC), a new generation of B-domain deleted recombinant factor VIII (BDDrFVIII) for treatment of haemophilia A: demonstration of safety, efficacy, and pharmacokinetic equivalence to full-length recombinant factor VIII. Haemophilia 15: 869-880, 2009.

24. Yang M, Liu R, Sheng J, Liao J, Wang Y, Pan E, Guo W, Pu Y and Yin L: Differential expression profiles of microRNAs as potential biomarkers for the early diagnosis of esophageal squamous cell carcinoma. Oncol Rep 29: 169-176, 2013.

25. Peng Y, Liu YM, Li LC, Wang LL and Wu XL: MicroRNA-338 inhibits growth, invasion and metastasis of gastric cancer by targeting NRP1 expression. PLoS One 9: e94422, 2014.

26. Xu H, Zhao L, Fang Q, Sun J, Zhang S, Zhan C, Liu S and Zhang Y: MiR-338-3p inhibits hepatocarcinoma cells and sensitizes these cells to sorafenib by targeting hypoxia-induced factor 1a. PLoS One 9: e115565, 2014.

27. Xue Q, Sun K, Deng HJ, Lei ST, Dong JQ and Li GX: MicroRNA$338-3 p$ inhibits colorectal carcinoma cell invasion and migration by targeting smoothened. Jpn J Clin Oncol 44: 13-21, 2014

28. Funato Y, Terabayashi T, Suenaga N, Seiki M, Takenawa T and Miki H: IRSp53/Eps8 complex is important for positive regulation of Rac and cancer cell motility/invasiveness. Cancer Res 64: 5237-5244, 2004

29. Liu PS, Jong TH, Maa MC and Leu TH: The interplay between Eps8 and IRSp53 contributes to Src-mediated transformation. Oncogene 29: 3977-3989, 2010.

30. Oh SY, Knelson EH, Blobe GC and Mythreye K: The type III TGF $\beta$ receptor regulates filopodia formation via a Cdc42mediated IRSp53-N-WASP interaction in epithelial cells. Biochem J 454: 79-89, 2013.

31. de Wit E and van Steensel B: Chromatin domains in higher eukaryotes: Insights from genome-wide mapping studies. Chromosoma 118: 25-36, 2009.

32. Moreno-Hagelsieb G: The power of operon rearrangements for predicting functional associations. Comput Struct Biotechnol J 13: 402-406, 2015.

33. Sampson VB, Yoo S, Kumar A, Vetter NS and Kolb EA: MicroRNAs and potential targets in osteosarcoma: Review. Front Pediatr 3: 69, 2015.

34. Stephens NA and Sparks LM: Resistance to the beneficial effects of exercise in type 2 diabetes: Are some individuals programmed to fail? J Clin Endocrinol Metab 100: 43-52, 2015.

35. Ling DJ, Chen ZS, Zhang YD, Liao QD, Feng JX, Zhang XY and Shi TS: MicroRNA-145 inhibits lung cancer cell metastasis. Mol Med Rep 11: 3108-3114, 2015.

36. Yan S, Liu G, Pei C, Chen W, Li P, Wang Q, Jin X, Zhu J, Wang M and Liu X: Inhibition of NADPH oxidase protects against metastasis of human lung cancer by decreasing microRNA-21. Anticancer Drugs 26: 388-398, 2015 .

37. Dou H, Wang Y, Su G and Zhao S: Decreased plasma let-7c and miR-152 as noninvasive biomarker for non-small-cell lung cancer. Int J Clin Exp Med 8: 9291-9298, 2015.

38. Li W, Wang Y, Zhang Q, Tang L, Liu X, Dai Y, Xiao L, Huang S, Chen L, Guo Z, et al: MicroRNA-486 as a biomarker for early diagnosis and recurrence of non-small cell lung cancer. PLoS One 10: $\mathrm{e} 0134220,2015$.

39. Zhao X, He X, Han X, Yu Y, Ye F, Chen Y, Hoang T, Xu X, Mi QS, Xin M, et al: MicroRNA-mediated control of oligodendrocyte differentiation. Neuron 65: 612-626, 2010. 
40. Saba R, Goodman CD, Huzarewich RL, Robertson C and Booth SA: A miRNA signature of prion induced neurodegeneration. PLoS One 3: e3652, 2008.

41. Ebrahimi-Barough S, Massumi M, Kouchesfahani HM and Ai J: Derivation of pre-oligodendrocytes from human endometrial stromal cells by using overexpression of microRNA 338. J Mol Neurosci 51: 337-343, 2013.

42. Ribeiro DM and Sonati MF: Regulation of human alpha-globin gene expression and alpha-thalassemia. Genet Mol Res 7: 1045-1053, 2008.

43. Tomomura M, Hasegawa Y, Hashikawa T, Tomomura A Yuzaki M, Furuichi T and Yano R: Differential expression and function of apoptosis-associated tyrosine kinase (AATYK) in the developing mouse brain. Brain Res Mol Brain Res 112: 103-112, 2003.
44. Vellon L, Menendez JA and Lupu R: AlphaVbeta3 integrin regulates heregulin (HRG)-induced cell proliferation and survival in breast cancer. Oncogene 24: 3759-3773, 2005.

45. Xia ZJ, Hu W, Wang YB, Zhou K and Sun GJ: Expression heterogeneity research of ITGB3 and BCL-2 in lung adenocarcinoma tissue and adenocarcinoma cell line. Asian Pac J Trop Med 7: 473-477, 2014.

46. Müller DW and Bosserhoff AK: Integrin beta 3 expression is regulated by let-7a miRNA in malignant melanoma. Oncogene 27: 6698-6706, 2008.

47. Sun L, Liu B, Lin Z, Yao Y, Chen Y, Li Y, Chen J, Yu D, Tang Z, Wang B, et al: MiR-320a acts as a prognostic factor and inhibits metastasis of salivary adenoid cystic carcinoma by targeting ITGB3. Mol Cancer 14: 96, 2015. 